PROCEEDINGS OF THE

AMERICAN MATHEMATICAL SOCIETY

Volume 125, Number 4, April 1997, Pages 1123-1129

S 0002-9939(97)03907-5

\title{
OPIAL'S INEQUALITY AND OSCILLATION OF 2ND ORDER EQUATIONS
}

\author{
R. C. BROWN AND D. B. HINTON
}

(Communicated by Hal L. Smith)

\begin{abstract}
For a second-order differential equation, we obtain from Opial's inequality lower bounds for the spacing between two zeros of a solution or between a zero of a solution and a zero of its derivative. These bounds are expressed in terms of antiderivatives of the potential, and in particular we derive some new Liapunov type inequalities from them.
\end{abstract}

\section{INTRODUCTION}

We consider the second-order linear differential equation

$$
y^{\prime \prime}+q(x) y=0, \quad a \leq x \leq b,
$$

where $q$ is a real measurable function on $[a, b]$ satisfying $\int_{a}^{b}|q(x)| d x<\infty$. Two problems of interest are: (i) obtain lower bounds for the spacing of zeros of a solution of (1.1), and (ii) obtain lower bounds for the spacing $\beta-\alpha$ where $y$ is a solution of (1.1) satisfying $y(\alpha)=y^{\prime}(\beta)=0$ or $y^{\prime}(\alpha)=y(\beta)=0$. Of particular interest in this paper is when $q$ is oscillatory and this behavior affects the bounds.

Our motivation for this work comes from a recent paper of Harris and Kong [3]. Two of their results [3, Theorems 2.1 and 2.2] state that if $y$ is a solution of (1.1) with no zeros in $(\alpha, \beta)$ and such that $y^{\prime}(\alpha)=y(\beta)=0$, then

$$
(\beta-\alpha) \max _{\alpha \leq x \leq \beta}\left|\int_{\alpha}^{x} q(t) d t\right|>1 .
$$

If instead $y(\alpha)=y^{\prime}(\beta)=0$, then

$$
(\beta-\alpha) \max _{\alpha \leq x \leq \beta}\left|\int_{x}^{\beta} q(t) d t\right|>1 .
$$

Finally, if there are no extreme values of $y$ in $(\alpha, \beta)$, then in either (1.2) or (1.3) the absolute value may be dropped. Their method of proof is to use Riccati equation techniques. Here, by using Opial's inequality, we prove several results which relate to problems (i) and (ii) above. In particular we obtain (1.2) and (1.3) as a consequence of Opial's inequality.

In section 2 we state the two forms of Opial's inequality that we employ. In section 3 we apply them to problem (ii) above, and in section 4 we apply them to

Received by the editors October 11, 1995.

1991 Mathematics Subject Classification. Primary 34C10; Secondary 34L05, 34L15.

(C) 1997 American Mathematical Society 
problem (i). Section 5 contains some further implications of section 4 . $\mathcal{L}(a, b)$ and $\mathcal{L}^{2}(a, b)$ denote the usual Lebesgue spaces.

\section{Two Opial TYPe INEQUALITIES}

A special case of an inequality obtained by Beesack and Das [1] is the following (see also [4, p. 119]).

Theorem A. If $f$ is absolutely continuous on $[a, b]$ with $f(a)=0$, and $s \in \mathcal{L}^{2}(a, b)$, then

$$
\int_{a}^{b} s(x)|f(x)|\left|f^{\prime}(x)\right| d x \leq \kappa \int_{a}^{b}\left|f^{\prime}(x)\right|^{2} d x
$$

where

$$
\kappa=\frac{1}{\sqrt{2}}\left(\int_{a}^{b} s(t)^{2}(t-a) d t\right)^{\frac{1}{2}}
$$

with equality if and only if $f \equiv 0$ (or $f$ is linear and $s$ is constant).

If we replace $f(a)=0$ in Theorem A by $f(b)=0$, then (2.1) holds where $\kappa$ in (2.2) is given by

$$
\kappa=\frac{1}{\sqrt{2}}\left(\int_{a}^{b} s(t)^{2}(b-t) d t\right)^{\frac{1}{2}} .
$$

We also use another Opial inequality which is a special case of a more general result due to Boyd [2].

Theorem B. If $f$ is absolutely continuous on $[a, b]$ with $f(a)=0$ or $f(b)=0$ and $1 \leq p \leq 2$, then

$$
\int_{a}^{b}|f(x)|^{p}\left|f^{\prime}(x)\right|^{p} d x \leq K(p)(b-a)\left(\int_{a}^{b}\left|f^{\prime}(x)\right|^{2} d x\right)^{p}
$$

where

$$
K(p)= \begin{cases}\frac{1}{2}, & p=1, \\ \frac{4}{\pi^{2}}, & p=2, \\ \frac{2-p}{2 p}\left(\frac{1}{p}\right)^{2 p-2} I^{-p}, & 1<p<2,\end{cases}
$$

with $I=\int_{0}^{1}\left\{1+\frac{2(p-1)}{2-p} t\right\}^{-2}\{1+(p-1) t\}^{\frac{1}{p}-1} d t$. For $p=1$, equality holds in (2.4) only for $f$ linear.

Theorem B has immediate application to the case where $f(a)=f(b)=0$. Choose $c=(a+b) / 2$ and apply $(2.4)$ to $[a, c]$ and $[c, b]$ and then add to obtain that

$$
\begin{aligned}
\int_{a}^{b}|f(x)|^{p}\left|f^{\prime}(x)\right|^{p} d x & \leq K(p)\left(\frac{b-a}{2}\right)\left\{\left(\int_{a}^{c}\left|f^{\prime}(x)\right|^{2} d x\right)^{p}+\left(\int_{c}^{b}\left|f^{\prime}(x)\right|^{2} d x\right)^{p}\right\} \\
& \leq K(p)\left(\frac{b-a}{2}\right)\left\{\int_{a}^{b}\left|f^{\prime}(x)\right|^{2} d x\right\}^{p} .
\end{aligned}
$$

For $p=1,(2.6)$ is strict unless $f$ is linear in each of the subintervals $[a, c]$ and $[c, b]$. 


\section{Disfocal problems}

Consider the differential equation

$$
y^{\prime \prime}+q(x) y=0, \quad a \leq x \leq b,
$$

where $q$ is real and $q \in \mathcal{L}(a, b)$.

Theorem 3.1. Suppose $y$ is a nontrivial solution of (3.1) which satisfies $y(a)=$ $y^{\prime}(b)=0$. Then

$$
1<2 \int_{a}^{b} Q(x)^{2}(x-a) d x
$$

where $Q(x)=\int_{x}^{b} q(t) d t$. If $y^{\prime}(a)=y(b)=0$, then

$$
1<2 \int_{a}^{b} Q(x)^{2}(b-x) d x
$$

where $Q(x)=\int_{a}^{x} q(t) d t$.

Proof. We first establish (3.2). Multiplying (3.1) by $y$ and integrating by parts gives

$$
\begin{aligned}
\int_{a}^{b} y^{\prime}(x)^{2} d x & =\int_{a}^{b} q(x) y(x)^{2} d x=\int_{a}^{b}-Q^{\prime}(x) y(x)^{2} d x \\
& =\int_{a}^{b} 2 Q(x) y(x) y^{\prime}(x) d x \\
& \leq 2 \int_{a}^{b}|Q(x)|\left|y(x) y^{\prime}(x)\right| d x \\
& <\frac{2}{\sqrt{2}}\left(\int_{a}^{b} Q(x)^{2}(x-a) d x\right)^{\frac{1}{2}} \int_{a}^{b} y^{\prime}(x)^{2} d x
\end{aligned}
$$

by (2.1) and (2.2) of Theorem A. The inequality is strict since $y$ linear implies $y \equiv 0$ as $y(a)=y^{\prime}(b)=0$. By cancelling $\int_{a}^{b} y^{\prime}(x)^{2} d x$ and squaring we obtain (3.2). The proof of (3.3) is similar using integration by parts and (2.1) of Theorem A and (2.3) instead of $(2.2)$.

By using the maximum of $|Q|$ on $[a, b]$ in (3.2) and (3.3), integrating, and then taking a square root we see that

$$
1<(b-a) \max _{a \leq x \leq b}\left|\int_{x}^{b} q(t) d t\right|
$$

when $y(a)=y^{\prime}(b)=0$, and

$$
1<(b-a) \max _{a \leq x \leq b}\left|\int_{a}^{x} q(t) d t\right|
$$

when $y^{\prime}(a)=y(b)=0$, which are the inequalities obtained by Harris and Kong. Note also that if $y$ has no extreme values in $(a, b)$, then $y y^{\prime}>0$ in the second line of (3.4). It follows that $Q(x) \leq Q_{+}(x) \equiv \max \{0, Q(x)\}$ and we can replace $Q$ by $Q_{+}$ in the derivation of (3.5). This means that the absolute value signs may be omitted in (3.5). With minor changes in the argument $\left(y y^{\prime}\right.$ is now negative on $\left.(a, b)\right)$, the same conclusion applies to (3.6) if $y$ has no extreme value on $(a, b)$. 
Results similar to Theorem 3.1 may be obtained by application of Boyd's theorem:

Theorem 3.2. Suppose $y$ is a nontrivial solution of (3.1) which satisfies $y(a)=$ $y^{\prime}(b)=0,1 \leq p \leq 2$, and $p^{\prime}$ is the conjugate index of $p$, i.e., $\frac{1}{p}+\frac{1}{p^{\prime}}=1$. Then

$$
1 \leq 2 K(p)^{\frac{1}{p}}(b-a)^{\frac{1}{p}}\left(\int_{a}^{b}|Q(x)|^{p^{\prime}} d x\right)^{\frac{1}{p^{\prime}}}
$$

where $Q(x)=\int_{x}^{b} q(t) d t$; if $y^{\prime}(a)=y(b)=0$, then (3.7) is true with $Q(x)=$ $\int_{a}^{x} q(t) d t$. In either case $K(p)$ is given by (2.5). For $p=1$ the inequality is strict. For $p=1$ the $p^{\prime}$ norm of $Q$ in (3.7) becomes $\max |Q(x)|, a \leq x \leq b$.

Proof. In the case $y(a)=y^{\prime}(b)=0$ from the proof of Theorem 3.1 we have that

$$
\int_{a}^{b} y^{\prime}(x)^{2} d x \leq 2 \int_{a}^{b}\left|Q(x)\|y(x)\| y^{\prime}(x)\right| d x .
$$

By application of Hölder's inequality and Theorem B to (3.8), we get that

$$
\begin{aligned}
\int_{a}^{b} y^{\prime}(x)^{2} d x & \leq 2\left(\int_{a}^{b}|Q(x)|^{p^{\prime}} d x\right)^{\frac{1}{p^{\prime}}}\left(\int_{a}^{b}\left|y(x) y^{\prime}(x)\right|^{p} d x\right)^{\frac{1}{p}} \\
& \leq 2 K(p)^{\frac{1}{p}}(b-a)^{\frac{1}{p}}\left(\int_{a}^{b}|Q(x)|^{p^{\prime}} d x\right)^{\frac{1}{p^{\prime}}} \int_{a}^{b} y^{\prime}(x)^{2} d x
\end{aligned}
$$

with strict inequality for $p=1$. Cancelling $\int_{a}^{b} y^{\prime}(x)^{2} d x$ yields (3.8). A similar argument yields (3.7) with $Q(x)=\int_{a}^{x} q(t) d t$ when $y^{\prime}(a)=y(b)=0$.

Note that for $p=1,(3.7)$ in the $y(a)=y^{\prime}(b)=0$ case is the same as (3.5) and in the $y^{\prime}(a)=y(b)$ case the same as (3.6).

Theorems 3.1 and 3.2 yield sufficient conditions for disfocality of (3.1), i.e., sufficient conditions so that there does not exist a nontrivial solution $y$ of (3.1) satisfying either $y(a)=y^{\prime}(b)=0$ or $y^{\prime}(a)=y(b)=0$.

\section{Disconjugacy CONDitions}

Application of (2.6) allows the use of an arbitrary anti-derivative $Q$ in the above arguments. From this we are able to prove new Lyapunov type inequalities.

Theorem 4.1. Suppose $y$ is a nontrivial solution of (3.1) which satisfies $y(a)=$ $y(b)=0,1 \leq p \leq 2$, and $Q^{\prime}(x)=q(x)$ on $[a, b]$. Then

$$
1 \leq 2 K(p)^{\frac{1}{p}}\left(\frac{b-a}{2}\right)^{\frac{1}{p}}\left(\int_{a}^{b}|Q(x)|^{p^{\prime}} d x\right)^{\frac{1}{p^{\prime}}}
$$

with $K(p)$ given by (2.5). For $p=1$ the inequality is strict.

Proof. As in the proof of Theorem 3.1, multiplying (3.1) by $y$ and integration by parts yields that

$$
\int_{a}^{b} y^{\prime}(x)^{2} d x=\int_{a}^{b} q(x) y(x)^{2} d x=-2 \int_{a}^{b} Q(x) y(x) y^{\prime}(x) d x .
$$


By application of Hölder's inequality and (2.6) to (4.2) we get that

$$
\begin{aligned}
\int_{a}^{b} y^{\prime}(x)^{2} d x & \leq 2\left(\int_{a}^{b}|Q(x)|^{p^{\prime}} d x\right)^{\frac{1}{p^{\prime}}}\left(\int_{a}^{b}\left|y(x) y^{\prime}(x)\right|^{p} d x\right)^{\frac{1}{p}} \\
& \leq 2 K(p)^{\frac{1}{p}}\left(\frac{b-a}{2}\right)^{\frac{1}{p}}\left(\int_{a}^{b}|Q(x)|^{p^{\prime}} d x\right)^{\frac{1}{p^{\prime}}} \int_{a}^{b} y^{\prime}(x)^{2} d x
\end{aligned}
$$

from which (4.1) follows. For $p=1$ the inequality is strict since a solution of (3.1) cannot be linear on each of the intervals $[a,(a+b) / 2],[(a+b) / 2, b]$ as this implies a discontinuity of $y^{\prime}$.

We examine (4.1) further in the cases $p=1,2$. For $p=1$, we take $Q(x)=$ $\int_{a}^{x} q(t) d t+\mu$. Then since $K(1)=\frac{1}{2}$,

$$
1<\left(\frac{b-a}{2}\right) \max _{a \leq x \leq b}\left|\int_{a}^{x} q(t) d t+\mu\right| .
$$

We now minimize (4.4) over $\mu$. This is seen to occur with $\mu=-(M+m) / 2$, where

$$
M=\max _{a \leq x \leq b} \int_{a}^{x} q(t) d t, \quad m=\min _{a \leq x \leq b} \int_{a}^{x} q(t) d t
$$

so that

$$
\inf _{\mu}\left(\max _{a \leq x \leq b}\left|\int_{a}^{x} q(t) d t+\mu\right|\right)=\frac{M-m}{2} .
$$

We have therefore the following corollary of Theorem 4.1.

Corollary 4.1. If $y$ is a nontrivial solution of (3.1) which satisfies $y(a)=y(b)=0$, then there exist $t_{1}$ and $t_{2}$ in $[a, b]$ such that

$$
\frac{4}{b-a}<\left|\int_{t_{1}}^{t_{2}} q(t) d t\right|
$$

For $p=2$, we square (4.1) to obtain that

$$
1 \leq \frac{8}{\pi^{2}}(b-a) \int_{a}^{b}\left(\int_{a}^{x} q(t) d t+\mu\right)^{2} d x .
$$

Again we minimize with respect to $\mu$ and find that

$$
\mu=-\frac{1}{(b-a)} \int_{a}^{b}\left(\int_{a}^{x} q(t) d t\right) d x .
$$

Using this value of $\mu$ yields another corollary.

Corollary 4.2. If $y$ is a nontrivial solution of (3.1) which satisfies $y(a)=y(b)=0$, then

$$
\frac{\pi^{2}}{8(b-a)} \leq \int_{a}^{b}\left(\int_{a}^{x} q(t) d t\right)^{2} d x-\frac{1}{b-a}\left(\int_{a}^{b}\left(\int_{a}^{x} q(t) d t\right) d x\right)^{2}
$$




\section{Applications}

First we consider the equation

$$
y^{\prime \prime}+\lambda(\cos k x) y=0, \quad 0 \leq x \leq B, \quad k>0 .
$$

Let $y$ be a nontrivial solution of (5.1) with $y(a)=y(b)=0$. By (4.5),

$$
\frac{4}{b-a}<\left|\int_{t_{1}}^{t_{2}} \lambda \cos k t d t\right|=\left|\frac{\lambda\left(\sin k t_{2}-\sin k t_{1}\right)}{k}\right| \leq \frac{2|\lambda|}{k}
$$

so that

$$
2 k<|\lambda|(b-a) \leq|\lambda| B .
$$

If we view (5.1) as an eigenvalue problem with indefinite weight function and boundary conditions $y(0)=y(\pi)=0$, then (5.2) says that a real eigenvalue $\lambda$ has $|\lambda|$ large if $k$ is large. More generally it is an inequality which gives a lower bound for the spacing of zeros in the form $(b-a)>2 k /|\lambda|$. With $\lambda=1,5$ and $k=1,5,10,20$ we have computed the first positive zero $b$ of the solution of (5.1) with $y(0)=0$, $y^{\prime}(0)=1$ using a MAPLE V subroutine. The results (rounded to 2 decimal places) are given below:

$$
\begin{array}{lcccc}
k & 1 & 5 & 10 & 20 \\
\lambda=1 & 7.37 & 22.25 & 44.4 & 88.85 \\
\lambda=5 & 1.77 & 4.33 & 8.88 & 17.77
\end{array}
$$

Thus (5.2) gives a lower bound for $b$ which for $k$ large is consistently slightly less than $50 \%$ of the true value. However this is still much better than the estimates for (5.1) obtainable by the usual Lyapunov inequality which for $\lambda=1, a=0$ is

$$
\frac{4}{b}<\int_{0}^{b}(\cos k x)_{+} d x,
$$

where + denotes the positive part of a function. This inequality gives a a lower bound for the spacing of zeros only of order $\sqrt{k}$ rather than $k$ as in (5.2).

Theorem 4.1 also allows for a counting of the number of zeros of a solution of (3.1). If a solution $y$ of (3.1) has consecutive zeros $a=a_{0}<a_{1}<\cdots<a_{n}=b$, then (4.1) yields that for $Q^{\prime}=q$,

$$
1 \leq 2 K(p)^{\frac{1}{p}}\left(\frac{a_{i}-a_{i-1}}{2}\right)^{\frac{1}{p}}\left(\int_{a_{i-1}}^{a_{i}}|Q(x)|^{p^{\prime}} d x\right)^{\frac{1}{p^{\prime}}} .
$$

If we sum (5.3) and apply Hölder's inequality we obtain that

$$
\begin{aligned}
n+1 & =\# \text { zeros of } y \text { in }[a, b] \\
& \leq 2 K(p)^{\frac{1}{p}}\left(\frac{b-a}{2}\right)^{\frac{1}{p}}\left(\int_{a}^{b}|Q(x)|^{p^{\prime}} d x\right)^{\frac{1}{p^{\prime}}}+1 .
\end{aligned}
$$

As a final application we show how Theorem 4.1 may be applied to yield a lower bound for the first eigenvalue $\lambda_{0}$ of

$$
-y^{\prime \prime}+q(x) y=\lambda y, \quad y(a)=y(b)=0 .
$$


Let $y$ be the eigenfunction of (5.4) corresponding to $\lambda_{0}$. Now choose $\mu<\lambda_{0}$ and let $Q^{\prime}(x)=q(x)-\mu$. Proceeding as in the proof of Theorem 4.1 yields that

$$
\begin{aligned}
\left(\lambda_{0}-\right. & \mu) \int_{a}^{b} y(x)^{2} d x=\int_{a}^{b} y^{\prime}(x)^{2} d x+\int_{a}^{b} Q^{\prime}(x) y(x)^{2} d x \\
& =\int_{a}^{b} y^{\prime}(x)^{2} d x-2 \int_{a}^{b} Q(x) y(x) y^{\prime}(x) d x \\
& \geq \int_{a}^{b} y^{\prime}(x)^{2} d x-2\left(\int_{a}^{b}|Q(x)|^{p^{\prime}} d x\right)^{\frac{1}{p}}\left(\int_{a}^{b}\left|y(x) y^{\prime}(x)\right|^{p} d x\right)^{\frac{1}{p}} .
\end{aligned}
$$

Since the first eigenvalue of $-y^{\prime \prime}=\lambda y, y(a)=y(b)=0$ is $\pi^{2} /(b-a)^{2}$, we have the Wirtinger type inequality

$$
\int_{a}^{b} y(x)^{2} d x \leq(b-a)^{2} \pi^{-2} \int_{a}^{b} y^{\prime}(x)^{2} d x .
$$

Using now (2.6) and (5.6) in (5.5) yields that

$$
\left(\lambda_{0}-\mu\right)(b-a)^{2} \pi^{-2} \geq 1-2 K(p)^{\frac{1}{p}}\left(\frac{b-a}{2}\right)^{\frac{1}{p}}\left(\int_{a}^{b}|Q(x)|^{p^{\prime}} d x\right)^{\frac{1}{p^{\prime}}},
$$

where $1 \leq p \leq 2$ and $K(p)$ is given by (2.5). Thus a lower bound for $\lambda_{0}$ is obtained.

The results given here may be applied to the equation

$$
\left(p(x) y^{\prime}\right)^{\prime}+q(x) y=0, \quad a \leq x \leq b,
$$

by a change of variable. If $y$ is a solution of (5.7) satsfying $y(a)=y(b)=0$, let $Y(t)=y(x)$ where $t=\int_{a}^{x} d s / p(s)$. Then $Y$ satisfies

$$
\frac{d^{2} Y}{d t^{2}}+R(t) Y(t)=0, \quad Y(0)=Y(T)=0
$$

where $R(t)=p(x) q(x)$ and $T=\int_{a}^{b} d s / p(s)$. Then Corollary 4.1 takes the form: if $y$ is a nontrivial solution of (5.7) satisfying $y(a)=0=y(b)$, then there exist $x_{1}, x_{2}$ such that

$$
\frac{4}{\int_{a}^{b} d s / p(s)}<\left|\int_{x_{1}}^{x_{2}} q(x) d x\right|
$$

\section{REFERENCES}

1. P. R. Beesack and K.M. Das, Extensions of Opial's inequality, Pacific J. Math. 26 (1968), 215-232. MR 39:385

2. D. W. Boyd, Best constants in a class of integral inequalities, Pacific J. Math. 30 (1969), 367-383. MR 40:2801

3. B. J. Harris and Q. Kong, On the oscillation of differential equations with an oscillatory coefficient, Trans. Amer. Math. Soc. 347 (1995), 1831-1839. MR 95h:34050

4. D. S. Mitrinović, J.E. Pěcarić, and A.M. Fink, Inequalities involving functions and their integrals and derivatives, Kluwer Academic Publishers, Dordrecht, 1991. MR 93m:26036

Department of Mathematics, University of Alabama, Tuscaloosa, Alabama 35487

E-mail address: dbrown@mathdept.as.ua.edu

Department of Mathematics, University of Tennessee, Knoxville, Tennessee 37996

E-mail address: hinton@novell.math.utk.edu 